\title{
Collective Motion of Secondary Electrons Visualized by Electron Holography
}

\author{
Daisuke Shindo ${ }^{1,2}$, Zentaro Akase ${ }^{1,2}$, and Hyun Soon Park ${ }^{1}$ \\ ${ }^{1 .}$ Institute of Multidisciplinary Research for Advanced Materials, Tohoku University, Sendai 980-8577, \\ Japan \\ 2.RIKEN Center for Emergent Matter Science (CEMS), Wako, Saitama 351-0198, Japan
}

The electromagnetic forces in various devices are mostly produced by the motion of electrons. Thus, it is of vital importance as well as significant interest to analyze the motion of electrons. After several trials, we have recently succeeded in visualizing the collective motion of secondary electrons by electron holography through the charging effect induced by electron irradiation on insulating biological materials.

The electron holography experiments were performed using HF-3300X and JEM-3000F transmission electron microscopes. In the simulation for the electric field analysis, the ELFIN software (ELF Corporation) was used for the simulation of the electric field, and ELF/BEAM software (ELF Corporation) was used for the trajectory analysis of secondary electrons. The simulation utilizing the software was performed on the basis of Maxwell's equations.

Figure 1 shows an experimental result of visualizing the collective motion of secondary electrons by electron holography. The specimen is a microfibril of sciatic nerve tissue with two branches. In the reconstructed amplitude images in Fig. 1, the red regions indicate the area where electric field fluctuates due to the motion of electrons. At the initial state of Fig. 1(a), the electric field variation with red color is not prominent. However, when the amount of electron irradiation is increased, red color regions are localized and the position of the region changes gradually between the two branches as indicated by arrows in Figs. 1(b) and 1(c). These results indicate that the collective motion of electrons can be detected through electric field variation and can be visualized through amplitude reconstruction process for holograms. In Figs. 2(a) and 2(b), two reconstructed phase images of a microfibril with different electron irradiation are presented. Two branches are colored blue. Taking into account the modulation of the reference wave due to the stray electric field, simulation of electric potential distribution was carried out. The simulated reconstructed-phase images are shown in Figs. 2(c) and 2(d) in which the electric potentials of the microfibril are assumed as $5 \mathrm{~V}$ and $15 \mathrm{~V}$, respectively. The simulation of Figs. 2(c) and 2(d) reproduces well the asymmetrical distribution of contour lines around the two branches observed in Figs. 2(a) and 2(b). Figure 3 indicates the simulation of the motion of secondary electrons. When the secondary electron with the initial energy $10 \mathrm{eV}$ is emitted from the left-side surface of the left branch as indicated by \#3, it leaves downwards and does not come back. On the other hand, the secondary electrons emitted from the right-side surface of the branch as indicated by \#4, it comes near the surface of the right branch due to its attractive force. Through these processes, the accumulation of the secondary electrons between the two branches is explained under the condition that the movement of the specimen is small [1].

Furthermore, it has been pointed out that under certain experimental conditions, the stationary orbits of secondary electrons could be located through amplitude reconstruction process. It has been also pointed out that visualizing the motion of electrons by electron holography is the so-called disturbance free observation [2]. Finally the present result of visualizing collective motion of electrons on the nanometer scale through electron holographic interferometry over the micrometer region is briefly discussed in terms of the field equations, i.e., Maxwell's (linear) equations and Einstein's gravitational (non-linear) equations. 


\section{References:}

[1] M Inoue, S Suzuki, Z Akase and D Shindo, J. Electron Microsc. 61 (2012) p. 217.

[2] D Shindo et al, J. Phys. Soc. Jpn. 78 (2009) 104802.
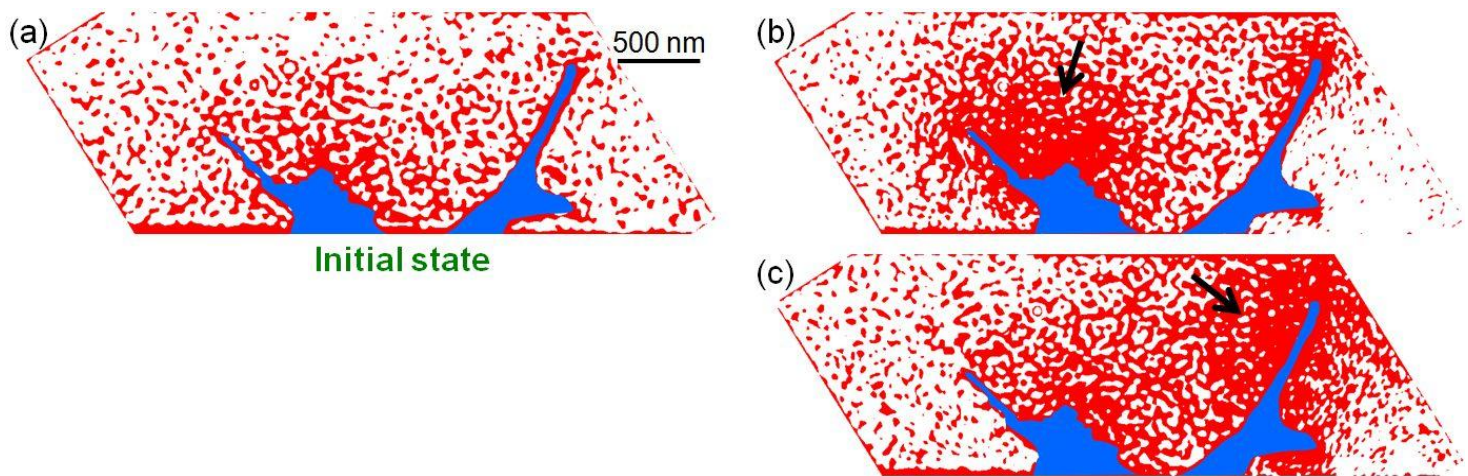

Figure 1. Binarized reconstructed-amplitude images (red and white) around a microfibril of sciatic nerve tissue (blue). The red regions indicate the area where electric field fluctuates due to the motion of electrons.

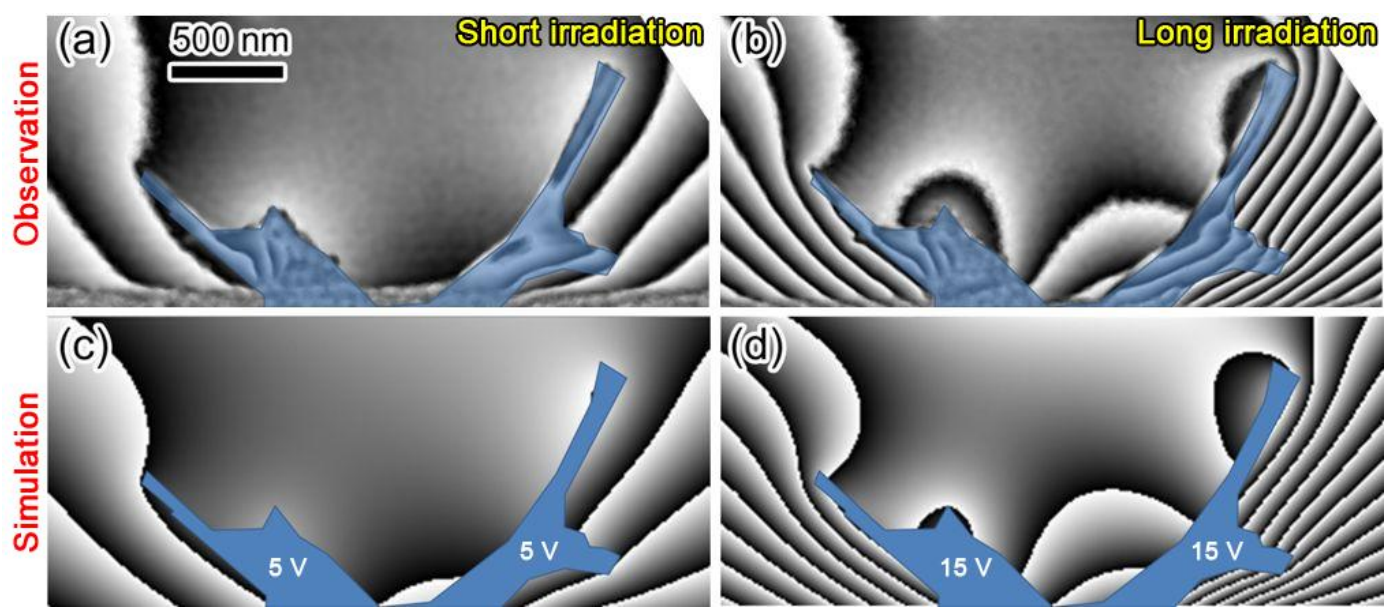

Figure 2. Averaged phase images of a microfibril under short (a) and long (b) irradiation. Simulation of reconstructed phase images assuming the electric potential of the microfibril at $5 \mathrm{~V}$ (c) and $15 \mathrm{~V}$ (d) taking into account the modulation of reference wave due to the electric stray field.

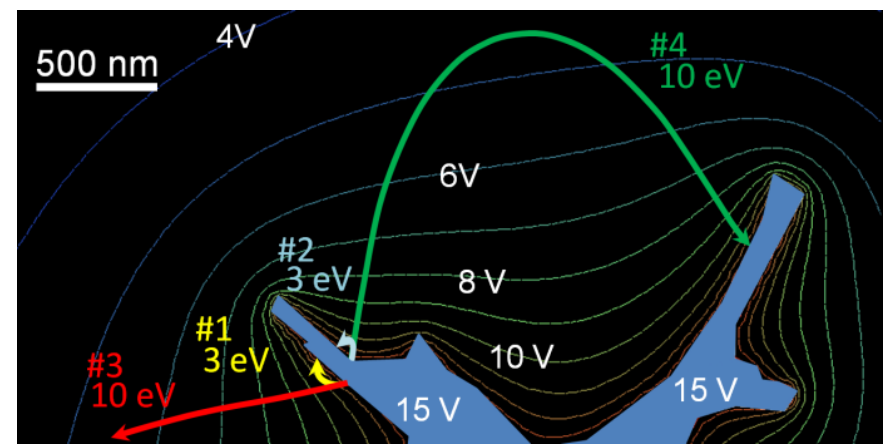

Figure 3. Simulation showing the trajectories of secondary electrons emitted from the left branch. Initial energies of secondary electrons are $3 \mathrm{eV}(\# 1$ and \#2) and $10 \mathrm{eV}(\# 3$ and \#4). 